\title{
Peculiarity of highly radiating multi-impurity seeded H-mode Plasmas on JET with ITER-like Wall
}

\author{
A. Huber ${ }^{1}$, M. Wischmeier ${ }^{2}$, M. Bernert ${ }^{2}$, S. Wiesen ${ }^{1}$, S. Glöggler ${ }^{2}$, S. Aleiferis ${ }^{3}$, S. Brezinsek ${ }^{1}$, \\ G. Calabro ${ }^{4}$, P. Carvalho ${ }^{5}$, V. Huber ${ }^{6}$, G. Sergienko ${ }^{1}$, E. R. Solano ${ }^{7}$, C. Giroud ${ }^{8}$, M. Groth ${ }^{9}$, \\ S. Jachmich ${ }^{10}$, Ch. Linsmeier ${ }^{1}$, G.F. Matthews ${ }^{8}$, A.G. Meigs ${ }^{8}$, Ph. Mertens ${ }^{1}$, M. Sertoli ${ }^{2}$, \\ S. Silburn ${ }^{8}$, G. Telesca ${ }^{11}$ \\ ${ }^{1}$ Forschungszentrum Jülich GmbH, Institut für Energie- und Klimaforschung - Plasmaphysik, Partner \\ of the Trilateral Euregio Cluster (TEC), 52425 Jülich, Germany \\ ${ }^{2}$ Max-Planck-Institut für Plasmaphysik, Boltzmannstraße 2, D-85748 Garching, Germany \\ ${ }^{3}$ NCSR 'Demokritos', 153 10, Agia Paraskevi Attikis, Greece \\ ${ }^{4}$ Department of Economics, Engineering, Society and Business Organization (DEIm), University of \\ Tuscia, Largo dell'Università snc, 01100 Viterbo, Italy \\ ${ }^{5}$ Instituto de Plasmas e Fusão Nuclear, Instituto Superior Técnico, Universidade de Lisboa, \\ Portugal, \\ ${ }^{6}$ Forschungszentrum Jülich GmbH, Supercomputing Centre, 52425 Jülich, Germany \\ ${ }^{7}$ Laboratorio Nacional de Fusión, CIEMAT, Madrid, Spain \\ ${ }^{8}$ CCFE, Culham Science Centre, Abingdon, OX14 3DB, UK \\ ${ }^{9}$ Aalto University, Association EURATOM-Tekes, P.O.Box 4100, 02015 Espoo, Finland \\ ${ }^{10}$ Laboratory for Plasma Physics, ERM/KMS, B-1000 Brussels, Belgium \\ ${ }^{11}$ Institute of Plasma Physics and Laser Microfusion, Warsaw, Poland \\ E-mail: $\underline{\text { A.Huber@,fz-juelich.de }}$
}

Keywords: power exhaust, radiation, impurities in plasmas, divertors, detachment, tokamaks PACS numbers: 52.55.Rk, 52.25.Vy, 52.40.Hf, 52.55.Fa

\begin{abstract}
On JET with fully metallic first wall, highly radiative conditions with $\mathrm{N}_{2}, \mathrm{Ne}$ and Ar as well as their mixture as radiators are approached in high density H-mode plasmas. The confinement increases from $\mathrm{H}_{98(\mathrm{y}, 2)}=0.65$ in unseeded pulses with $\gamma_{\mathrm{rad}} \sim 30 \%$ to a value of $\mathrm{H}_{98(\mathrm{y}, 2)}=0.75$ at $\gamma_{\mathrm{rad}} \sim 50 \%$ with $\mathrm{N}_{2}$ injection. A degradation of the pedestal profile is compensated by steeper core $\mathrm{n}_{\mathrm{e}}$ and $\mathrm{T}_{\mathrm{e}}$ profiles. Further increase of $\gamma_{\mathrm{rad}}$ with increase of the $\mathrm{N}_{2}$ seeding rate leads to a moderate confinement degradation which can be avoided by applying of combined impurity seeding. The enhancement of the plasma performance for the radiation fractions beyond $55 \%$ with the maximum value of $\mathrm{H}_{98(\mathrm{y}, 2)}=0.78$ is reached with combined $\mathrm{N}_{2}+\mathrm{Ne}$ and $\mathrm{N}_{2}+\mathrm{Ar}$ impurity injections. The observed intense, strongly localized radiation at the X-point inside the confined plasma in the scenarios with the highest radiated power fraction is interlinked to complete divertor detachment. In the JET-ILW, the X-point radiation is stable, reproducible and reversible.
\end{abstract}

\section{Introduction}

The development of plasma scenarios with high fractions of radiated power through impurity seeding is essential for the safe operation of future fusion devices with a burning plasma such as ITER and DEMO where perpendicular divertor target loads should be kept below $5-10 \mathrm{MWm}^{-2}[1,2]$ to avoid divertor damage. At the same time, the electron temperature at the target needs to be low enough to restrict the erosion of the divertor to acceptable levels. However, radiative power losses within the confined plasma can affect the plasma confinement 
and fuel dilution as well as the discharge stability. Therefore, it is very important to understand underlying physical processes in highly radiative discharges to provide the recipe for controlled seeding with a reasonable reduction in the power flux and target temperature while minimizing the impact on the confined plasma.

Consequently, the selection of an extrinsic impurity for fusion devices is based on its radiative characteristics in the regions of divertor and main plasma as well as on their influence on plasma confinement under high divertor radiation conditions. Also the interaction of the seeded impurities with the plasma-facing components (PFCs), which affects the tritium processing plant, should be taken into account. In the present tokamak experiments nitrogen is favoured as divertor radiator since it is more efficiently compressed than $\mathrm{Ne}$. At the same time $\mathrm{N}_{2}$ provides adequately localized divertor radiation $[3,4,5]$. However, nitrogen is chemically active and can cause the formation of ammonia. During the ITER operation with the Deuterium-Tritium fuel mix and $\mathrm{N}_{2}$ impurity seeding, a tritiated ammonia $\left(\mathrm{NT}_{3}\right)$ will be produced [6]. The amount of $\mathrm{NT}_{3}$ produced may be significant and could have an impact on the exhaust treatment, which will affect the design of the ITER tritium plant. In contrast to the experimental observations, simulations of ITER plasmas at high performance [7] show that compression for $\mathrm{N}_{2}$ and $\mathrm{Ne}$ will be similar. In addition, $\mathrm{Ne}$ is not chemically reactive, which makes it an attractive candidate as a divertor radiator in ITER. In addition to $\mathrm{N}_{2}$ and $\mathrm{Ne}$, argon is considered also as a further candidate for simultaneous improvement of core and divertor radiation, when some increased radiation in the main chamber is requested. It is assumed that adequate divertor compression for Ar can be achieved.

Impurities, such as neon $(\mathrm{Ne})$, nitrogen $\left(\mathrm{N}_{2}\right)$ and argon (Ar) are commonly used in experiments with strongly radiative divertor on many tokamaks $[8,9,10]$. On the other hand, interpretation studies on mixed seed discharges in tokamaks have not been performed very often $[11,12]$. Therefore, further investigations on mixed seeding experiments must be performed to develop scenarios with a reasonable energy confinement simultaneously with a high level of radiation.

\section{Impurity seeding in high-density H-mode plasmas}

\section{$2.1 \mathrm{~N}_{2}$ seeding in high density H-mode plasmas}

After the first wall of JET was upgraded to an ITER-like wall (ILW) [13], the level of carbon was reduced at least by a factor of 10 [14] leading to a strong reduction of the divertor radiation in the fully metallic machine.

Nitrogen proves to be an appropriate replacement for carbon as a divertor radiator thanks to its maximum radiative efficiency at low temperatures, $\mathrm{T}_{\mathrm{e}} \sim 10-20 \mathrm{eV}$ [15]. Dedicated H-mode 
nitrogen seeding experiments have been carried out at JET with the ITER-like wall (JET-ILW) to study the impact of seeding with diverse impurities on the energy confinement. In addition, the influence of different impurity species on the balance between radiation in the divertor region and in the main chamber has been investigated. High density Type I ELMy H-mode plasmas at $\mathrm{B}_{\mathrm{T}}=2.7 \mathrm{~T}, \mathrm{I}_{\mathrm{p}}=2.5 \mathrm{MA}\left(\mathrm{q}_{95}=3.3\right)$ at Greenwald density fraction $\mathrm{f}_{\mathrm{GW}}\left(=<\mathrm{n}_{\mathrm{e}}>/ \mathrm{n}_{\mathrm{e}, \mathrm{GW}}\right)$ up to $85 \%$ in low-triangularity magnetic equilibria $(\delta=0.22)$ with both strike points on the lower vertical targets have been examined. Thereby, the Greenwald density limit $\mathrm{n}_{\mathrm{e}, \mathrm{GW}}\left[10^{20} \mathrm{~m}^{-3}\right]=\mathrm{I}_{\mathrm{p}}$ $[\mathrm{MA}] /\left(\pi \mathrm{a}^{2}\right)$ and $\mathrm{a}$ is the plasma minor radius in $\mathrm{m}[16]$. Respective scans have been performed over a deuterium fuelling range of $2 \times 10^{22} \mathrm{el} / \mathrm{s} \div 6.5 \times 10^{22} \mathrm{el} / \mathrm{s}$ and an impurity seeding range of $2 \times 10^{22} \mathrm{el} / \mathrm{s} \div 1.3 \times 10^{23}$ el/s with an auxiliary heating power of 18MW. Fuelling and seeding rate are given in this contribution in unit of electrons per second $(\mathrm{el} / \mathrm{s})$ due to the complete ionisation of the injected gas flow. The emission signal of beryllium in the outer divertor (Be II $527 \mathrm{~nm}$ ) with high temporal resolution $(10 \mathrm{kHz})$ represents the behaviour of ELMs. Opposite to an unseeded plasma (not shown in
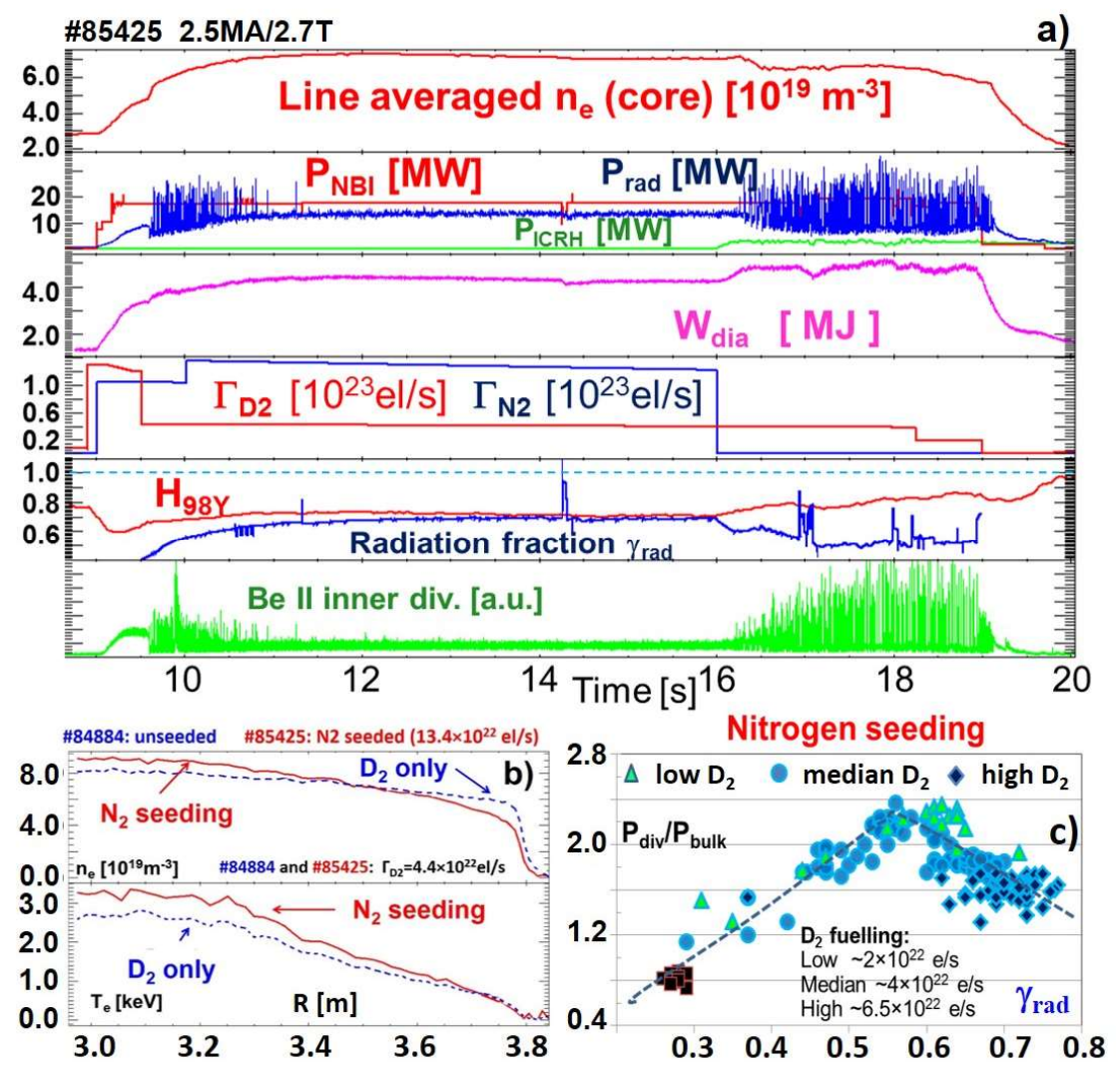

Fig. 1 a) Time traces of central line averaged $n_{e}$, NBI and ICRH heating power as well as total radiated power, plasma stored energy, $\mathrm{D}_{2}$-fuelling and $\mathrm{N}_{2}$-seeding waveforms, $\gamma_{\mathrm{rad}}$ and $\mathrm{H}_{98(\mathrm{y}, 2)}$-factor, BeII fast emission signal in the inner divertor of a $\mathrm{N}_{2}$-seeded high-density H-mode plasma on JET-ILW b) comparison of $\mathrm{n}_{\mathrm{e}}$ and $T_{e}$ profiles for nitrogen $\mathrm{c}$ ) the ratio of the divertor radiation to the bulk radiation.

the figure), the

radiation scenario with $\mathrm{N}_{2}$ seeding shows small energy ELMs directly after the L-H transition, with a fast transition at $10.8 \mathrm{~s}$ from a Type I ELMy to a stable and stationary ELM-free H-mode phase.

The $\mathrm{N}_{2}$ gas has been injected into the private flux region of the outer divertor leg with seeding rate of $\Gamma_{\mathrm{N} 2} \approx 13.4 \times 10^{22} \mathrm{el} / \mathrm{s}$. The ELM-free H-mode phase demonstrates cold pedestal $\left(\mathrm{T}_{\mathrm{e}}{ }^{\text {ped }} \approx 300 \mathrm{eV}\right)$ and some recovery of the energy confinement (Fig. 1a). During this ELM free H- 
mode, the so-called M-mode [17] with plasma performance between L-mode and H-mode (medium confinement), the radiation fraction reaches the value of $\gamma_{\mathrm{rad}} \sim 75 \%$. This mode can typically be observed during the operation with the input power close to the L-H power threshold or just right after an L-H transition. During the M-mode phase the characteristic oscillation can be seen in the fast divertor $\mathrm{D}_{\alpha}$ emissions as well as in the signals of a poloidal Mirnov coil.

In the unseeded plasma, the global confinement was significantly degraded at higher fuelling rate required to reach a high density close to Greenwald limit [16]. In comparison with the unseeded pulse, the ion effective charge $Z_{\text {eff }}$ increases during the $\mathrm{N}_{2}$ seeding by $\approx 50 \%$ from 1.1 to 1.7 (increase of $\Delta \mathrm{Z}_{\mathrm{eff}}=0.6$ ). The radiation fraction in this pulse reaches its peak-value of about $75 \%$. Considerable energy confinement recovery is observed with $\mathrm{N}_{2}$ seeding. It increases from a confinement factor of $\mathrm{H}_{98(\mathrm{y}, 2)}=0.65$ in the pulse with $\mathrm{D}_{2}$ fuelling only to a factor of $\mathrm{H}_{98(\mathrm{y}, 2)}=0.75$. Figure $1 \mathrm{~b}$ shows profiles of the electron temperature $\left(\mathrm{T}_{\mathrm{e}}\right)$ and density $\left(\mathrm{n}_{\mathrm{e}}\right)$ measured by the High Resolution Thomson Scattering system [18] in unseeded as well as seeded plasmas. Contrary to the case of unseeded plasma, the electron density ne in the nitrogen seeded plasmas decreases in the edge region and increases in the plasma core correspondingly, demonstrating a more significantly peaked behaviour (see Fig.1b). This steepening is explained using ASTRA-TGLF simulations by a stabilizing effect on the ITG modes through dilution of the main ion density [19]. On the other hand, $\mathrm{T}_{\mathrm{e}}$ increases with $\mathrm{N}_{2}$ seeding over the entire profile. Consequently, the impurity seeding strongly affects the particle transport and confinement, whereby possibly increasing the plasma stored energy. In contrast to these experiments, the $\mathrm{N}_{2}$ seeding in JETILW in high triangularity geometry demonstrates an enhanced energy confinement due to an improved pedestal [9]. Also the plasma performance improvement with nitrogen is observed on ASDEX Upgrade where the improvement is clearly related to the improved pedestal performance [20].

Fig.1c shows the ratio of the divertor radiation to the bulk radiation in the plasmas with injected $\mathrm{N}_{2}$. In this contribution, we use the definition of the divertor radiation, Pdiv, as the total radiation which is emitted below a height of $Z \leq-1.0 \mathrm{~m}$ (for definition of the coordinate $Z$ see Fig.3), and $\mathrm{P}_{\mathrm{rad}}{ }^{\text {bulk }}=\mathrm{P}_{\mathrm{rad}}{ }^{\text {tot }}-\mathrm{P}_{\mathrm{rad}}{ }^{\text {div. }}$. This definition is commonly used on many existing machines $[21,22]$. The reason for using this definition is as follows. The determination of the total power radiated below the X-point is not trivial, since the spatial resolution of the bolometer system on JET is not sufficient to determine whether radiation originates from inside or outside the separatrix. This is particularly problematic when emission radiation profiles have a peak in the vicinity of the X-point. For the characterisation of the radiation distribution, we are using here the ratio of radiation fractions in divertor and main chamber $\left(\mathrm{P}_{\mathrm{rad}}{ }^{\mathrm{div}} / \mathrm{P}_{\mathrm{rad}}{ }^{\text {bulk }}\right)$. This evaluated ratio, as well 
as $\gamma_{\mathrm{rad}}{ }^{\mathrm{div}}$, does not demonstrate an obvious dependence on $\mathrm{D}_{2}$-fueling in $\mathrm{N}_{2}$-seeded, type-I ELMy $\mathrm{H}$-mode pulses. The fraction of the divertor radiation increases initially with increase of the radiation fraction and reach the maximal value at $\gamma_{\mathrm{rad}} \sim 55-60 \%$. Additional increase of $\gamma_{\mathrm{rad}}$ leads to a reduction of the $\mathrm{P}_{\mathrm{rad}}{ }^{\mathrm{div}} / \mathrm{P}_{\mathrm{rad}}$ bulk indicating some level of saturation in the divertor radiation.

\subsection{Ne and Ar seeding in high density H-mode plasmas}

In addition to $\mathrm{N}_{2}$ seeded scenarios, high radiation plasma scenarios with $\mathrm{Ne}$ and Ar seeding were investigated at JET. Neon is an auspicious candidate as a radiating impurity for plasma scenarios in the future reactors radiating effectively in the plasma edge at temperatures of $\approx 50$ $\mathrm{eV}[15]$.

As is discussed in [3], Ar and Ne seeding increased the total radiation fraction up to a level of $\gamma_{\mathrm{rad}} \approx 63 \%$ which is below the highest achieved radiated power fraction in $\mathrm{N}_{2}$ seeded plasmas (75\%). The radiation distribution inclined somewhat more biased towards the plasma core with radiation fraction of $\gamma_{\mathrm{rad}}{ }^{\mathrm{div}} \approx 0.5$ in the divertor area, as one might expect from the electron temperature dependence of the radiative cooling efficiency function [15]. Contrary to N2 seeding which leads to a significant improvement of the plasma energy confinement, the $\mathrm{Ar}$ injection causes only a moderate enhancement. Ne and Ar radiate as well in the pedestal region and main plasma (respectively) decreasing the power entering the scrape-off-layer (SOL) and consequently lowering the pedestal confinement $[3,5]$. It was observed that Ar seeding prompts an $\mathrm{H}-\mathrm{L}$ transition at radiation fraction $\gamma_{\mathrm{rad}}$ of $\approx 60 \%[3]$.

As demonstrated in detail in [3], the discharges with Ne injection showed beyond the radiation level of $\gamma_{\mathrm{rad}} \approx 50 \%$ an ELM-free phase (M-mode, as defined above) with a dithering between Hand L-mode (sequences of H-L-H transitions). During the transient L-mode periods of these transitions, the $\gamma_{\text {rad }}$ rises up to $95 \%$, whereas the electron density is going down in the edge and in the core. The radiation profiles demonstrate divergent behaviours during the transition cycles as reported in [3]: during the L-mode period, the radiation pattern is located around the X-point with strong contributions in the inner and outer SOL; during the H-mode phase (or M-mode), the radiation profile is peaked in the zone above the $\mathrm{X}$-point within the separatrix. During the ELM-free H-mode (or M-mode) phases with Ne seeding, an improvement of the confinement factor up to $\mathrm{H}_{98(\mathrm{y}, 2)} \approx 0.75$ at $\gamma_{\mathrm{rad}} \sim 50 \%$ is observed. At the same time, the ion effective charge $\mathrm{Z}_{\text {eff }}$ is increasing by $\approx 80 \%$ (increase of $\Delta Z_{\text {eff }}=0.9$ to the value of $Z_{\text {eff }}=2.0$ for the duration of the seeding $)$ in Ne seeded plasmas in comparison with unseeded plasma $\left(Z_{\mathrm{eff}}=1.1\right)$.

\subsection{Combined $N_{2}$ and Ne seeding in H-mode plasmas}


A detailed study of the synergistic effects of combined impurity injections is required to predict the practicality of such highly radiating scenarios to future fusion devices. Fig. 2 shows the time traces of the plasma parameters in the plasma discharge with a combined gas seeding of $\mathrm{N}_{2}$ and Ne. The discharge $\left(\mathrm{I}_{\mathrm{P}} / \mathrm{B}_{\mathrm{T}}=2.5 \mathrm{MA} / 2.7 \mathrm{~T}\right)$ is carried out in a vertical target divertor geometry at a low triangularity configuration $\left(\delta_{\mathrm{av}} \approx 0.23\right)$. NBI and ICRH heating powers were 18.5 MW and 4.5MW respectively to maintain the plasma stored energy ( $\left.\mathrm{W}_{\mathrm{dia}}\right)$ of 4.8 MJ. The nitrogen and neon are injected at the

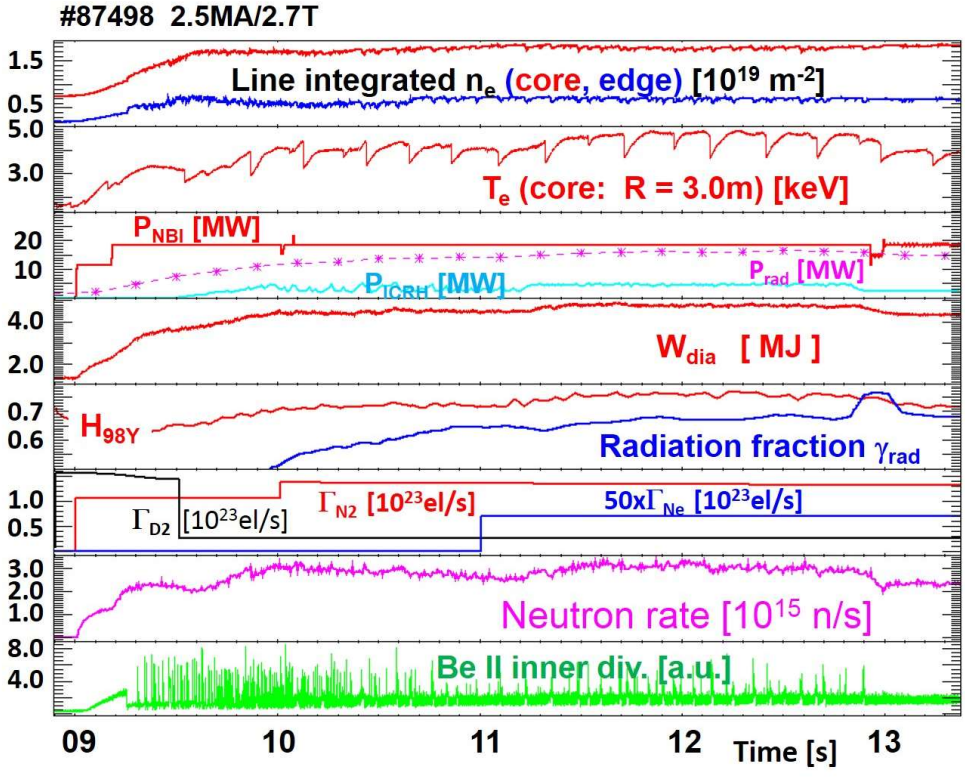

Fig.2: Time traces of combined $\mathrm{N}_{2}+\mathrm{Ne}$ seeded ELMy $\mathrm{H}$-mode discharge in JET-ILW (\#87498). From top: line-integrated core and edge electron density measured by interferometer, $\mathrm{T}_{\mathrm{e}}$ at $\mathrm{R}=3.0 \mathrm{~m}$ measured by the $\mathrm{ECE}$ diagnostic, NBI and ICRH heating power as well as total radiated power, diamagnetic stored energy, $\mathrm{H}_{98(\mathrm{y}, 2)}$ confinement factor and $\gamma_{\mathrm{rad}}, \mathrm{D}_{2-}$ fuelling and $\mathrm{N}_{2}$ - and $\mathrm{Ne}$-seeding waveforms, neutron rate, $\mathrm{Be}$ II fast emission signal in the outer divertor.

\section{horizontal tiles into the}

private flux region (PFR). The $\mathrm{N}_{2}$ injection is started during the ramp-up phase in order to avoid the high heat loads on the divertor and to prevent $\mathrm{W}$ sputtering and its accumulation during this phase. The $\mathrm{N}_{2}$ injection provokes the increase of the radiation fraction which reaches a quasistationary level of about $60 \%$ prior to the Ne injection phase $\left(Z_{\mathrm{eff}}=2.0\right)$. Before the Ne seeding, the divertor is in a fully detached state and the normalized confinement following the ITER physics base scaling is around $\mathrm{H}_{98(\mathrm{y}, 2)}=0.71$ at about $80 \%$ of the Greenwald density. No significant heat flux is measured at the divertor targets. From $t=11 \mathrm{~s}$, the small Ne gas puff is injected into the divertor (injection rate of $1.4 \times 10^{21} \mathrm{el} / \mathrm{s}$ ). By adding a small amount of seeded $\mathrm{Ne}$ (seeded fraction of $\Gamma_{\mathrm{Ne}} / \Gamma_{\mathrm{N} 2} \approx 1 \%$ ) to the $\mathrm{N}_{2}$ puff, particle confinement and transport could be significantly improved. $\mathrm{T}_{\mathrm{e}}$ rises in the plasma core without pedestal alteration. The electron temperature in the plasma core was measured by the electron cyclotron emission (ECE) diagnostic [23]. $Z_{\text {eff }}$ increases during the combined seedings and reaches the value of 2.35 at $\mathrm{t}=13.0 \mathrm{~s}$. Additionally, fig. 2 shows an improvement of the plasma stored energy during the combined seeding phase. As a result, applying a combination of nitrogen and neon gas seeding (11.4-13s), good confinement $\left(\mathrm{H}_{98(\mathrm{y}, 2)}=0.78\right)$ with high radiation fraction $\left(\gamma_{\mathrm{rad}}=0.7\right)$ was reached in high density Type-I ELMy H-mode. 
Also a slight increase of the neutron rate is observed during the phase with combined $\mathrm{N}_{2}$ and Ne seeding. In the plasmas studied here, the neutrons are primarily created by the interaction of the NBI beams with the thermal deuterons. This result is in agreement with the experimental observations reported in [19] where the neutron rate increases with increased neon puffing in the experiments under experimental conditions similar to those reported here.

Fig. 3 shows the radiation distributions and the vertical Z-averaged radiation distributions for H-mode plasmas with $\mathrm{N}_{2}$ seeding only as well as for combined $\mathrm{N}_{2}$ and $\mathrm{Ne}$ seeding. The tomographically reconstructed radiation distribution shows intense radiation in the vicinity of the X-point for $\mathrm{N}_{2}$ seeded plasmas as well as for plasmas with combined impurities injection. This observation is consistent with the statement given in [24] where it is reported that for conductive parallel heat flow, the plasma radiation in the scrape-off layer will peak in the region of the largest parallel temperature gradients. The largest parallel $\mathrm{T}_{\mathrm{e}}$-gradients are expected close to the divertor and around the X-point. It should be noted, however, that it is very likely that the radiation-associated heat sink leads to the formation of a steep parallel temperature gradient. The radiation distribution extends from the X-point peak its 'wing-like' pattern upwards along the separatrix towards the outside and inside mid-plane positions. As can be seen, it decreases strongly as one departs from the divertor. These findings are in agreement with the expected parallel $\mathrm{T}_{\mathrm{e}}$-gradient along the scrape-off layer. The electron temperature measured by Langmuir probes in the vicinity of the outer strike point during the combined impurity seeding reduces to $5 \mathrm{eV}$, which is the sensitivity level of the Langmuir probes. The inner target reaches these low temperatures earlier confirming the typical divertor asymmetry: a colder inner divertor region in comparison to the outer divertor leg. At the same time the total inner and outer saturation current exhibits a strong drop during the seeding, indicating that both targets are completely detached.

Adding a small amount of seeded $\mathrm{Ne}$ (seeded fraction of $\Gamma_{\mathrm{Ne}} / \Gamma_{\mathrm{N} 2} \approx 1 \%$ ) increased strongly the radiation around X-point (Fig.3) without altering the radiation in the main chamber as shown in the vertical Z-averaged radiation distribution (fig.3a). The increased seeded fraction to $\Gamma_{\mathrm{Ne}}$ $/ \Gamma_{\mathrm{N} 2} \approx 4 \%$ at the middle seeding rate $\left(\Gamma_{\mathrm{N} 2} \approx 7.6 \times 10^{22} \mathrm{el} / \mathrm{s}\right)$ increased significantly the $\mathrm{X}$-point radiation and only moderately the core radiation (fig.3b) without visible impact on the energy confinement. 


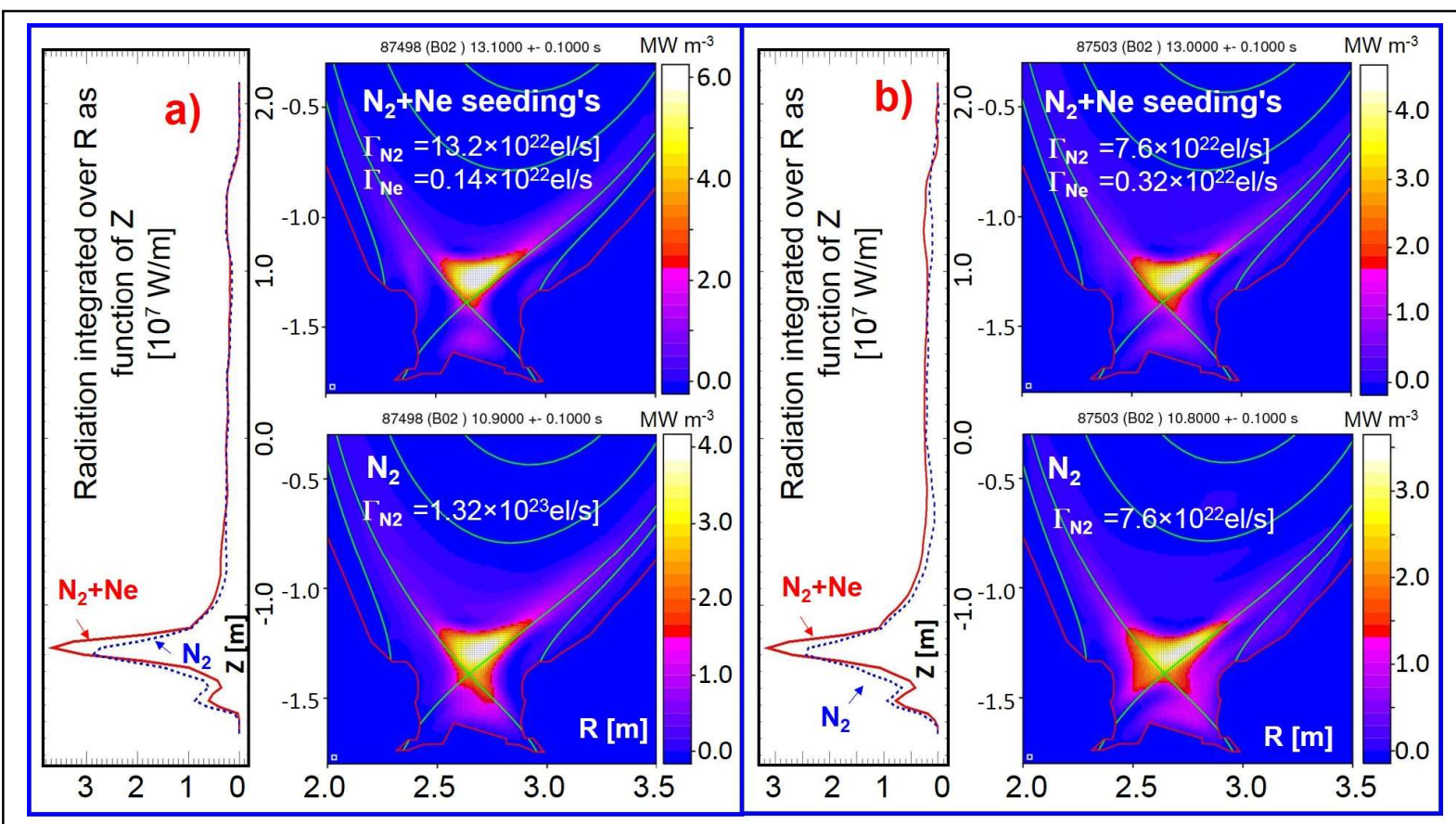

Fig.3 The vertical Z-averaged radiation distribution as well as tomographic reconstructions of the bolometric data for $\mathrm{N}_{2}$ seeded as well as for the simultaneous $\mathrm{N}_{2}$ and $\mathrm{Ne}$ injections. Figures a) and b) represent the cases with different impurity seeding rates as well as with different $\Gamma_{\mathrm{Ne}} / \Gamma_{\mathrm{N} 2}$ ratios. During the combined impurity seeding, $\mathrm{Z}_{\mathrm{eff}}$ reaches the values of 2.32 (Fig.3b) and 2.35 (Fig.3a) (Fig.3b).

\subsection{Combined $N_{2}$ and Ar seeding in H-mode plasmas}

The radiation enhancement in the SOL region is more pronounced for the lower- $\mathrm{Z}$ atoms such as $\mathrm{N}_{2}$ and Ne compared to Ar. The cooling efficiency of Ar reaches peak values at $\mathrm{T}_{\mathrm{e}} \sim 20 \mathrm{eV}$ and $\sim 200 \mathrm{eV}$ compared with that of $\mathrm{N}$ and $\mathrm{Ne}$ ions, which has a peak at $20-50 \mathrm{eV}$ [15]. It is worth mentioning that the cooling efficiency of $\mathrm{Ar}$ is higher than that of $\mathrm{Ne}$ and $\mathrm{N}_{2}$. Correspondingly, argon reveals the highest radiative efficiency for the electron temperature range expected in the divertor region. Due to its high radiation losses in the core plasma, however, no high argon concentrations are permitted unless a very high compression in the divertor can be achieved [10]. Combined gas seeding of $\mathrm{N}_{2}$ and $\mathrm{Ar}$ gas seeding was investigated in high density H-mode discharges. Fig.4 shows the radiation distributions and the vertical Zaveraged radiation distributions for $\mathrm{H}$-mode plasmas with $\mathrm{N}_{2}$ seeding only as well as for combined $\mathrm{N}_{2}$ and Ar injection. The nitrogen and Ar are injected (see Fig.4a) at constant seeding rates of $\Gamma_{\mathrm{N} 2} \approx 1.04 \times 10^{23} \mathrm{el} / \mathrm{s}\left(8.2 \times 10^{22} \mathrm{el} / \mathrm{s}\right.$ in Fig. $\left.4 \mathrm{~b}\right)$ and $\Gamma_{\mathrm{Ar}} \approx 0.41 \times 10^{22} \mathrm{el} / \mathrm{s}\left(1.1 \times 10^{22} \mathrm{el} / \mathrm{s}\right.$ in Fig.4b) which corresponds to the impurity seeded mixed fraction of $\Gamma_{\mathrm{Ar}_{\mathrm{r}}} / \Gamma_{\mathrm{N} 2} \approx 4 \%(13 \%$ in Fig.4b). For both cases, with $\mathrm{N}_{2}$ only seeding $\left(\mathrm{Z}_{\mathrm{eff}} \approx 1.85\right)$ as well as with combined $\mathrm{N}_{2}$ and $\mathrm{Ar}$ 


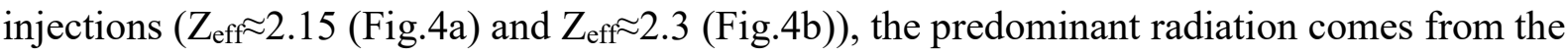
confined area and is located in a region near the X-point. Nevertheless, in contrast to combined

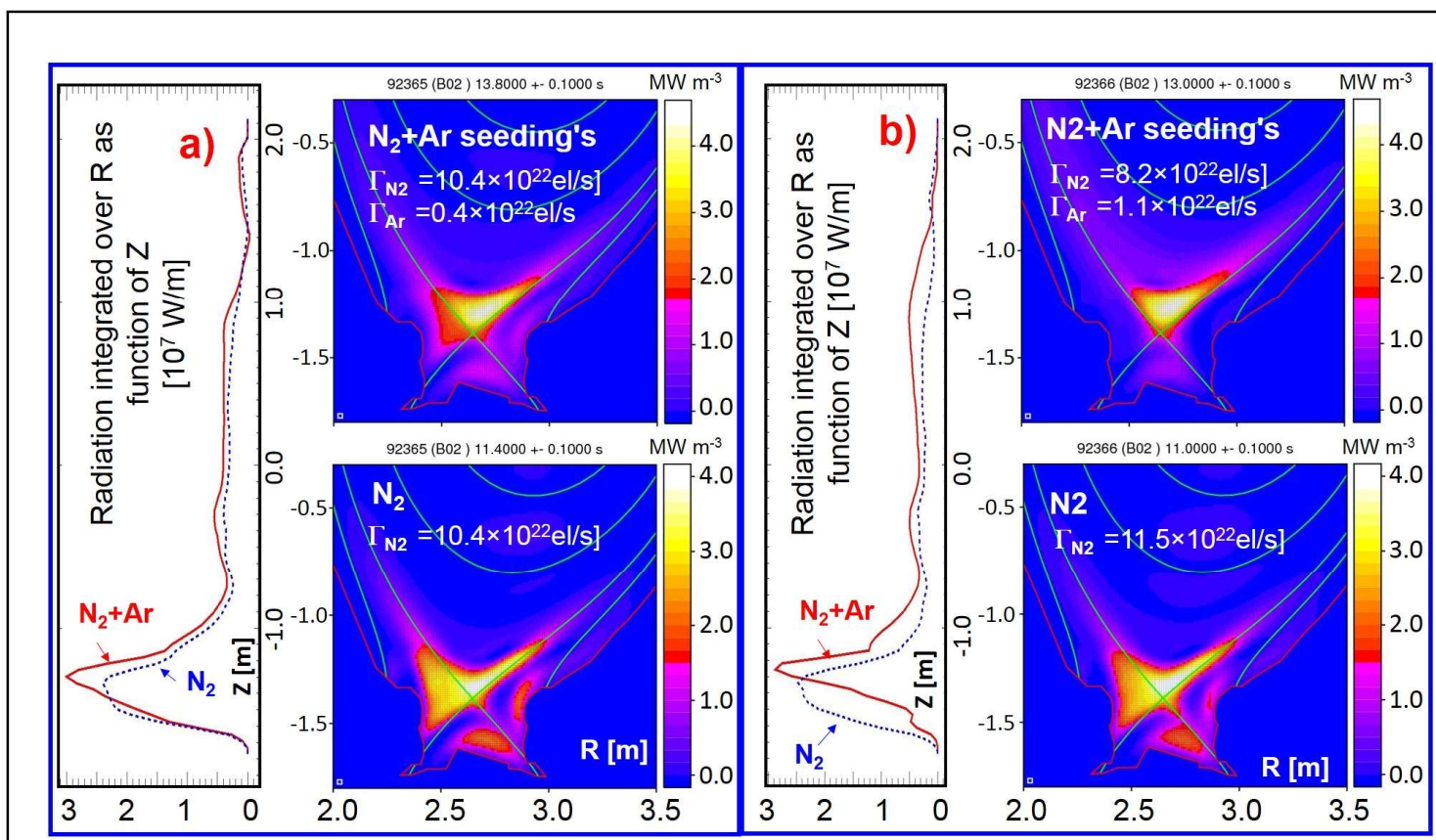

Fig.4 The vertical Z-averaged radiation distribution as well as tomographic reconstructions of the bolometric data for $\mathrm{N}_{2}$ seeded and for the simultaneous $\mathrm{N}_{2}$ and $\mathrm{Ar}$ injections. Figures a) and $\mathrm{b}$ ) represent the examples with different impurity seeding rates as well as with different $\Gamma_{\mathrm{Ar}} / \Gamma_{\mathrm{N} 2}$ ratios.

impurity puffing, the radiation in the inner divertor is clearly visible for the plasma with $\mathrm{N}_{2}$ cooling alone. From the X-point peak, the radiation distribution with combined impurity injections extends much strongly the 'wings-like' pattern upwards along the separatrix towards the inside and outside mid-plane positions. These radiation patterns reveal a clear in-out asymmetry. It could be explained by the strong poloidal asymmetry of the distribution of injected impurity ions. The impurity poloidal distribution in the plasma edge was intensively studied in [25] where the simulation with B2SOLPS5.2 transport code predicts a strong poloidal asymmetry. The asymmetry is explained by neoclassical effects in a plasma with strong gradients [25]. A significant increase of the radiation in the main plasma ( $\left.\mathrm{P}_{\text {rad }}{ }^{\text {bulk }}\right)$ can be clearly seen in the vertical Z-averaged radiation distributions (Fig.4a,b): the increase of about $50 \%$ from 7.2 MW to 10.9 MW for the case shown in Fig. 4a and the rise of $\approx 75 \%$ from $6.2 \mathrm{MW}$ to 10.8 MW in the case of Fig. 4b. These vertical distributions (Fig.4a,b) do not show any significant improvement in the peak divertor radiation. It should be noted here that the radiation pattern in the private flux region in Fig. 4 is an artefact, which are arising from the problem of non-perfect covering of the JET bolometry system of the corner region in the outer divertor leg. On the other hand, the location of maximum radiation moves to the direction of the main chamber. These results are consistent with tomographic reconstructions of the radiation 
distributions: in the combined seeding scenario $\left(\mathrm{N}_{2}+\mathrm{Ar}\right)$ radiation "spills over" into the area above the X-point inside of the separatrix.

\section{Confinement in high-density highly radiative H-mode plasma scenarios}

With regard to future fusion devices, the effect of various seed impurities on the plasma confinement needs to be investigated. Fig.5 shows the confinement factor $\mathrm{H}_{98(\mathrm{y}, 2)}$, measured during the experiments with three seeded impurity species $\mathrm{N}_{2}, \mathrm{Ne}$ and $\mathrm{Ar}$, as a function of radiated power fraction. The plasma performance in the $\mathrm{N}_{2}$ seeded plasmas was analysed over a deuterium fuelling range of $2 \times 10^{22} \mathrm{el} / \mathrm{s} \div 6.5 \times 10^{22} \mathrm{el} / \mathrm{s}$ and an impurity seeding range of $2 \times 10^{22}$ $\mathrm{el} / \mathrm{s} \div 1.3 \times 10^{23} \mathrm{el} / \mathrm{s}$. The confinement increases from $\mathrm{H}_{98(\mathrm{y}, 2)}=0.65$ in unseeded pulses with $\gamma_{\mathrm{rad}} \sim 30 \%$ to a value of $\mathrm{H}_{98(\mathrm{y}, 2)}=0.75$ at $\gamma_{\mathrm{rad}} \sim 50 \%$ with $\mathrm{N}_{2}$ injection.

As it is discussed in section 2.1, the $\mathrm{N}_{2}$ seeding significantly affects the particle confinement and transport increasing the energy stored in the plasma: the degraded electron density pedestal is accompanied by an $n_{e}$ increase in the plasma core as well as an increase of the electron temperature $\mathrm{T}_{\mathrm{e}}$ in the core. Further increase of $\gamma_{\text {rad }}$ with increase of the $\mathrm{N}_{2}$ seeding rate leads to a moderate confinement degradation. Beyond

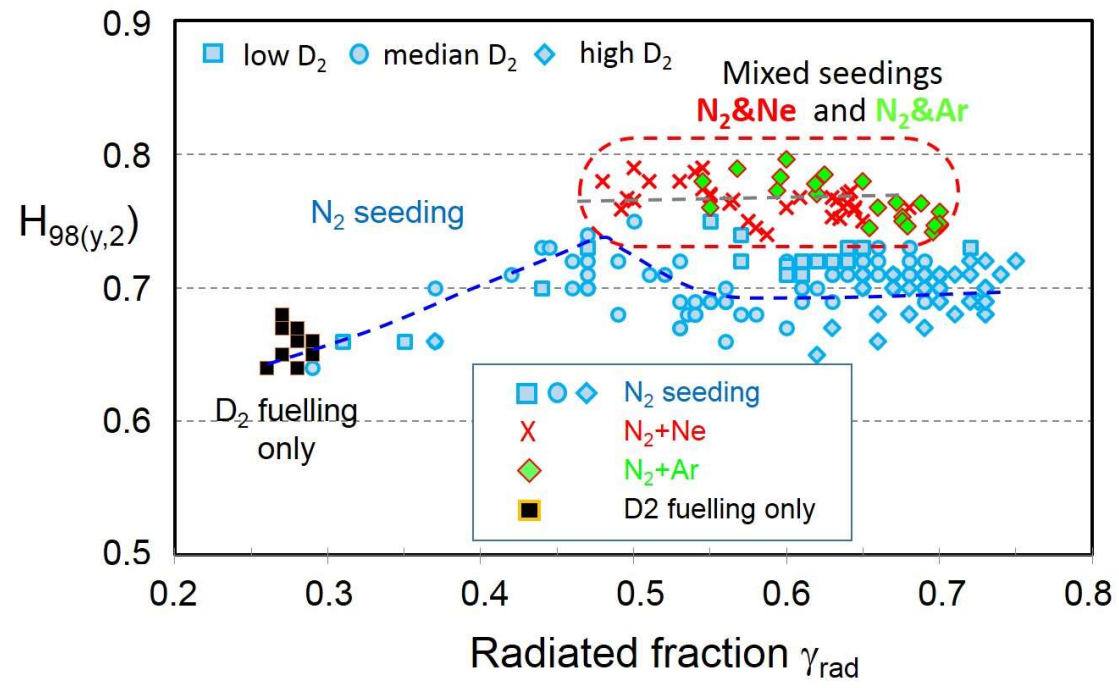

Fig. $5 \mathrm{H}_{98(\mathrm{y}, 2)}$ as a function of radiated power fraction. Improved confinement for combined $\mathrm{N}_{2}+\mathrm{Ne}$ as well as $\mathrm{N}_{2}+$ Ar radiators is approached in high density, highly radiative $\mathrm{H}$-mode plasma scenarios.

$\gamma_{\mathrm{rad}} \sim 55 \%$, the energy

confinement scaling factor remains almost constant at $\approx 0.7$.

The confinement improvement during the $\mathrm{N}_{2}$ seeding in JET-ILW is in contrast to findings seen in JET-C with a carbon divertor, where a reduction of $\mathrm{H}_{98(\mathrm{y}, 2)}$ was always observed during impurity seeding [26,27]. Comparison of the thermal energy confinement for the unseeded discharges as well as for three seeding scenario cases is shown in fig.5. This figure demonstrates the further enhancement of the plasma performance for the radiation fractions beyond $55 \%$. The maximum value of $\mathrm{H}_{98(\mathrm{y}, 2)}=0.78$, reached at combined $\mathrm{N}_{2}+\mathrm{Ne}$ and $\mathrm{N}_{2}+\mathrm{Ar}$ impurity injections, is lower than required for ITER. It should be noted, however, that the unseeded discharges have 
already a low confinement factor $\mathrm{H}_{98(\mathrm{y} .2)}$ of about 0.65 . Therefore, the impurity seeded ELMy H-mode discharges allow operation at higher densities compared to unseeded reference discharges, with good confinement or at least without a strong degradation of the confinement, as illustrated in Fig.5.

\section{Conclusion}

To find the optimum proportion of multiple seeded impurity species $\left(\mathrm{Ne}, \mathrm{Ar}, \mathrm{N}_{2}\right)$ compatible with a high confinement, dedicated H-mode impurity seeding experiments with different mixtures of seeded impurities have been performed in JET-ILW. We investigated how different mixtures influence the radiation patterns, radiation distributions as well as the confinement in the divertor and in the plasma core. The nitrogen causes a considerable energy confinement recovery from a confinement factor of $\mathrm{H}_{98(\mathrm{y}, 2)} \approx 0.65$ in the discharge with $\mathrm{D}_{2}$ fuelling only to a factor of $\mathrm{H}_{98(\mathrm{y}, 2)} \approx 0.75$ at $\gamma_{\mathrm{rad}} \sim 50 \%$. Further increase of $\gamma_{\mathrm{rad}}$ with increase of the $\mathrm{N}_{2}$ seeding rate leads, however, to a moderate confinement degradation. These losses of the plasma performance for the radiation fractions beyond $55 \%$ can be recovered by applying of combined $\mathrm{N}_{2}+\mathrm{Ne}$ and $\mathrm{N}_{2}+\mathrm{Ar}$ impurity injections. The confinement factor increased from 0.7 with $\mathrm{N}_{2}$ seeding to the value of $\mathrm{H}_{98(\mathrm{y}, 2)}=0.78\left(\mathrm{~N}_{2}+\mathrm{Ne}\right.$ or $\mathrm{N}_{2}+\mathrm{Ar}$ seeding). By adding a small amount of seeded $\mathrm{Ne}$ (seeded fraction of $\Gamma_{\mathrm{Ne}} / \Gamma_{\mathrm{N} 2} \approx 1 \%$ ) to the $\mathrm{N}_{2}$ puff, particle confinement and transport is significantly affected. $T_{e}$ rises in the plasma core without pedestal alteration. As a result, the

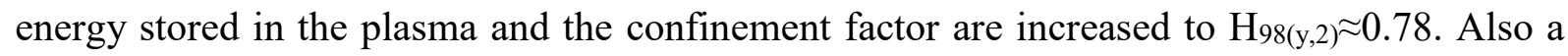
good confinement of $\mathrm{H}_{98(\mathrm{y}, 2)} \approx 0.77$ at a seeded fraction of $\Gamma_{\mathrm{Ar}} / \Gamma_{\mathrm{N} 2} \approx 4 \%$ has been demonstrated regardless of significant radiation from inside the separatrix.

There is an intense radiation in the vicinity of the $\mathrm{X}$-point for $\mathrm{N}_{2}$ seeded plasmas as well as for plasmas with injection of combined impurities. The small seeded fraction of $\Gamma_{\mathrm{Ne}} / \Gamma_{\mathrm{N} 2} \approx 1 \%$ increased strongly the radiation around X-point without altering the radiation in the main chamber. On the other hand, the increased seeded fraction to $\Gamma_{\mathrm{Ne}} / \Gamma_{\mathrm{N} 2} \approx 4 \%$ improved notably the X-point radiation and only moderately the radiation in the plasma core. The radiation distribution with combined $\mathrm{N}_{2}+$ Ar impurity injections extends much strongly the 'wings-like' pattern upwards along the separatrix towards the inside and outside mid-plane positions. At this impurity mix, a pronounced increase of the radiation in the main plasma has been observed. These experiments with combined impurity seeding show that an increase of the radiation inside the separatrix has no impact on the energy confinement. Additionally, it is demonstrated that the X-point radiation in the JET-ILW is stable, reproducible and reversible. 
Based on the result of this contribution, a mix of impurities could be considered for ITER and DEMO, a low-Z impurities for divertor radiation and a medium- $Z$ impurities for radiation in the confined region to optimize the heat flux removal to the first wall by radiation in the divertor region and in the main plasma chamber. However, it should be noted that in contrary to JET, ITER will operate very close to the L-H power threshold. Therefore, an increase of the pedestal radiation seems to bear higher risks for triggering in ITER the back transition from $\mathrm{H}$ to $\mathrm{L}$ mode. This might hinder the mid-Z radiator applicability. In ITER the "natural" radiation from intrinsic impurities, He and Bremsstrahlung is expected to be around $30 \%$ of the total loss power from the plasma, $P_{\text {loss }}=P_{\text {heat }}-\mathrm{dW} / \mathrm{dt}$, and thus no further reduction of the power flux across the separatrix is required in the standard ITER scenarios. But if ITER scenarios would require lower target heat loads for risk mitigation, then the mid-Z may be needed. On the other hand, the DEMO fusion reactor has to dissipate $\approx 70 \%$ of the total heating power (of around $450 \mathrm{MW}$ [28]), to be radiated inside the separatrix and, therefore, a mid-Z impurity is required [5].

\section{Acknowledgements}

This work has been carried out within the framework of the EUROfusion Consortium and has received funding from the Euratom research and training programme 2014-2018 and 2019-2020 under grant agreement No 633053. The views and opinions expressed herein do not necessarily reflect those of the European Commission.

\section{References}

[1] Loarte A et al 2007 Nucl. Fusion 47 S203-S263

[2] Raffray A R et al 2010 Fusion Eng. Des. 85 93-108

[3] Huber A et al Impact of Strong Impurity Seeding on the Radiation Losses in JET with ITER-like Wall, in: Proc. of the 41th EPS Conference on Controlled Fusion and Plasma Physics, Berlin, 2014), 2014 http://ocs.ciemat.es/EPS2014PAP/pdf/ P1.031.pdf, P1.031.

[4] Bernert M et al 2017 Nucl. Mater. Energy 12 111-118

[5] Wischmeier M et al $2015 \mathrm{~J}$. Nucl. Mater. 463 22-29 ISSN 0022-3115

[6] Oberkofler M et al 2013 J. Nucl. Mater. 438 (2013) S258-S261

[7] Kukushkin A S et al 2011 Fusion Eng. Des. 86 2865, https://doi.org/10.1016/j. fusengdes.2011.06.009.

[8] Reinke M L et al 2011 J. Nucl. Mater. 415 S340-S344

[9] Giroud C et al 2013 Nucl.Fusion 53113025

[10] Kallenbach A et al 2013 Plasma Phys. Control. Fusion 55124041

[11] Asakura N et al 2009 Nucl. Fusion 49115010 (2009).

[12] Kallenbach A et al 2012 Nucl. Fusion 52122003

[13] Matthews G F et al 2011 Phys. Scr. T145 014001.

[14] Brezinsek S et al 2013 J. Nucl. Mater. 438 S303-S308

[15] Post D et al 1995 Phys. Plasmas 22328

[16] Greenwald M 2002 Plasma Phys. Control. Fusion 44 R27

[17] Solano E R et al 2017 Nuclear Fusion 57022021

[18] Pasqualotto R et al 2004 Rev. Sci. Instrum. 753891

[19] S. Gloeggler to be published in Nucl. Fusion

[20] Schweinzer J et al 2011 Nuclear Fusion 51113003. 
[21] Fuchs J C et al., J. Nucl. Mat. 290-293 (2001) 525

[22] Ingesson L C et al., Radiation in Impurity-Seeded Discharges in the JET MkI, MkIIA \& MkII GB Divertors (2002)EFDA-JET-CP(02)01/11

[23] de la Luna E et al 2004 Rev. Sci. Instrum. 753831

[24] Pitcher C S and Stangeby P C 1997 Plasma Phys. Control. Fusion 39779

[25] Rozhansky V et al. Nucl. Fusion 55 (2015) 073017 (8pp)

[26] Maddison G. et al Nuclear Fusion 51 (2011) 042001.

[27] Giroud C et al 2012 Nucl. Fusion 52063022.

[28] Zohm H. et al 2013 Nucl. Fusion 53073019 